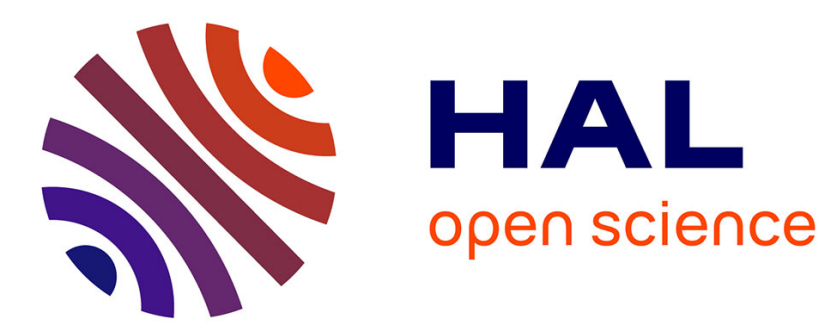

\title{
Caractérisation du timbre des sons complexes.I. Analyse multidimensionnelle
}

\author{
S. Donnadieu, S. Mcadams, S. Winsberg
}

\section{To cite this version:}

S. Donnadieu, S. Mcadams, S. Winsberg. Caractérisation du timbre des sons complexes.I. Analyse multidimensionnelle. Journal de Physique IV Proceedings, 1994, 04 (C5), pp.C5-593-C5-596. 10.1051/jp4:19945126 . jpa-00252802

\section{HAL Id: jpa-00252802 https://hal.science/jpa-00252802}

Submitted on 1 Jan 1994

HAL is a multi-disciplinary open access archive for the deposit and dissemination of scientific research documents, whether they are published or not. The documents may come from teaching and research institutions in France or abroad, or from public or private research centers.
L'archive ouverte pluridisciplinaire HAL, est destinée au dépôt et à la diffusion de documents scientifiques de niveau recherche, publiés ou non, émanant des établissements d'enseignement et de recherche français ou étrangers, des laboratoires publics ou privés. 


\title{
Caractérisation du timbre des sons complexes. I. Analyse multidimensionnelle
}

\author{
S. DONNADIEU ${ }^{* * *}$, S. McADAMS ${ }^{* * *}$ et S. WINSBERG ${ }^{* *}$ \\ * Laboratoire de Psychologie Expérimentale, CNRS, Université René Descartes, 28 rue Serpente, \\ 75006 Paris, France \\ ** IRCAM, 1 place Stravinsky, 75004 Paris, France
}

\begin{abstract}
Multidimensional scaling techniques are used to create a geometric representation of the mental organization of perceptual or conceptual relations. In this study they are used to explore the perceptual structure among different timbres ("timbre space"). The distance between any two timbres corresponds to their degree of perceptual dissimilarity. 98 listeners, musicians and nonmusicians of diverses nationalities, were asked to judge the degree of dissimilarity between all pairs of a set of 18 synthesized timbres equalized for pitch, loudness, and subjective duration. A multidimensionsal scaling analysis with the program CLASCAL revealed a space including three common dimensions shared by all the timbres as well as a set of weights that indicate the existence of perceptual features specific to certain timbres ("specificities"). Two of these dimensions seem to represent qualitatively the "brightness" and "attack quality" of the tones. Classes of listeners were determined by from the correlational structure of the raw dissimilarity matrices and weights are computed for each class. There was no systematic difference between the five classes associated with the musical training of the subjects.
\end{abstract}

\section{INTRODUCTION}

Les nombreux débats sur le timbre posent, au travers d'énoncés divers, la difficulté de sa définition. Ainsi l'American Standards Association propose de définir celui-ci comme "l'attribut de la sensation auditive suivant lequel un auditeur peut différencier deux sons présentés dans les mêmes conditions et ayant la même sonie et la même hauteur" [1]. De plus, le timbre en tant qu'attribut multidimensionnel de la sensation auditive, ne peut se mesurer sur un seul continuum (faible-intense ou grave-aigu). Le problème a donc été de mettre en évidence le nombre de dimensions qu'il possède. Les techniques d'échelles multidimensionnelles se sont avérées fructueuses dans l'étude des relations perceptives entre différents timbres. Ces 'modèles permettent de déterminer dans quel espace euclidien, c'est-à-dire selon combien de dimensions, différents timbres peuvent être rangés de façon à ce que les distances entre ceux-ci respectent le plus possible les jugements de dissemblance établis par des auditeurs. Le degré de dissemblance entre deux stimuli sera donc représenté par la distance métrique entre les points correspondants, ce qui suppose l'existence d'une relation monotone entre les jugements des sujets et les points représentés dans un tel espace. Les différentes dimensions de l'espace multidimensionnel sont alors interprétées comme étant les dimensions perceptives du timbre. Néanmoins, le timbre se définirait non seulement par un certain nombre de dimensions continues mais aussi selon des traits spécifiques qui les caractériseraient les uns des autres. Certains modèles hybrides permettent de rendre compte de ces spécificités susceptibles d'influencer la perception des relations entretenues par différents timbres. Krumhansl [2] a ainsi dégagé trois dimensions perceptives et des spécificités propres à certains timbres. Les trois axes correspondaient qualitativement à 1) "l'enveloppe temporelle" (qualité de l'attaque); 2) le "flux spectral"; 3) "l'enveloppe spectrale" (brillance). Cette étude cherche à reproduire un tel espace à partir de jugements effectués par des auditeurs dont l'expérience musicale varie du non musicien au musicien professionnel. Le modèle CLASCAL [3] permet de rendre compte des différences entre classes de sujets dans la détermination du poids des dimensions et de l'importance des "spécificités". Si les sujets qui se regroupent dans des classes, leurs différences de 
pondération peuvent permettre de dégager diverses stratégies dans l'évaluation des jugements de dissemblance, éventuellement entre musiciens et non musiciens si la pertinence perceptive des dimensions et/ou spécificités est distinctes pour ces deux types d'auditeurs.

\section{METHODE}

\subsection{Stimuli}

Dix huit parmi les 21 timbres créés sur un synthétiseur Yamaha TX802 déjà employés par Krumhansl [1] ont été utilisés. Douze sont des imitations d'instruments traditionnels et six sont des hybrides entre ces instruments. La hauteur, la durée subjective et la sonie de tous ces sons ont été égalisées. Toutes les 153 paires de sons différentes qu'il est possible de constituer avec les dix huit timbres ont été présentées aux sujets. Chaque paire n'est présentée qu'une fois et l'ordre de présentation des timbres à l'intérieur de celle-ci est aléatoire.

\subsection{Procédure}

Les stimuli sont présentés de façon diotique par l'intermédiaire d'un casque AKG Monitor K 240. Le sujet est placé dans une salle insonorisée, face à l'ordinateur. Après lecture de la consigne, les 18 timbres lui sont présentés dans un ordre aléatoire. Ensuite, il effectue 15 essais d'entraînement choisis au hasard parmi les 153 paires. Pour chacune de ces paires le sujet doit effectuer un jugement du degré de dissemblance des sons perçus à l'aide d'une échelle en 9 points allant du plus similaire au plus dissemblable. Suit alors les 153 paires, présentées dans un ordre aléatoire. Lors d'un essai, le sujet peut écouter chaque paire autant de fois qu'il le souhaite avant de donner son jugement définitif.

\subsection{Sujets} musiciens.

Les 98 sujets comprenaient 22 musiciens professionnels, 22 étudiants en musique et 54 non

\section{RESULTATS}

\subsection{Analyse de clusters}

Cette analyse permet de dégager une éventuelle structure de groupement des 98 sujets en fonction de la corrélation entre leurs jugements de dissemblance pour toutes les paires de timbres afin de dégager certains sujets dont le comportement serait très différent de la majorité. L'analyse révéla un sous-groupe de 10 sujets qui semblait s'éloigner de tous les autres. Parmi ces derniers 9 étaient des non musiciens. Le seul musicien était corrélé négativement à l'ensemble des autres sujets ce qui suggère qu'il aurait exécuté la tâche inverse (jugement de similarité). Les données de ces 10 personnes ont été exclues des analyses qui suivent.

\subsection{Analyse multidimensionnelle}

\subsubsection{Détermination du nombre de classes}

Une simulation Monté Carlo regroupe les 88 sujets en 5 classes. Les deux premières classes contiennent la majorité des sujets (classe $1=35$, classe $2=43$ ). La classe 3 ne comportait qu'un seul sujet non musicien. La classe 4 contient autant de musiciens que de non musiciens et la classe 5 ne contient que des musiciens.

\subsubsection{Détermination du nombre de dimensions}

Une analyse pour un nombre de dimensions allant de 2 à 8 a été effectuée pour 5 classes de sujets avec et sans spécificités. Une statistique du log de vraissemblance indique que la solution tend vers 6 dimensions sans spécificités ou 3 dimensions avec spécificités. Une structure en 3 dimensions ayant été obtenue pour les données des musiciens analysés séparément, nous avons opté pour une solution tridimensionnelle avec spécificités. 


\subsection{Analyse qualitative des dimensions}

Le Tableau I présente les corrélations effectuées entre les trois dimensions et spécificités de l'espace de Krumhansl (KWW) avec les dimensions et spécificités de notre espace (dim 1-3).

Tableau I. Corrélations entre l'espace de Krumhansl et l'espace en 3 dimensions avec spécificités. * p<.05.

\begin{tabular}{|c|c|c|c|c|}
\cline { 2 - 5 } \multicolumn{1}{c|}{} & $\operatorname{dim} \mathbf{1}$ & $\operatorname{dim} 2$ & $\operatorname{dim} \mathbf{3}$ & specificités \\
\hline KWW 1 Attaque & $.976^{*}$ & .094 & .272 & \\
\hline KWW 2 Flux & -.332 & -.198 & .244 & \\
\hline KWW 3 Brillance & -.006 & $-.955^{*}$ & -.072 & \\
\hline KWW specificités & & & & $.585^{*}$ \\
\hline
\end{tabular}

Les première et troisième dimensions de notre espace semblent correspondre qualitativement aux dimensions "qualité d'attaque" et "brillance" de l'espace de Krumhansl. Les spécificités sont également corrélées aux spécificités de Krumhansl. En revanche, la dimension "flux spectral" n'explique aucune des 3 dimensions de notre espace. Afin de retrouver cette 3ème dimension nous avons effectué une rotation procrustienne qui ajuste les deux structures le plus possible par des opérations de rotation et de mise en échelle. Le tableau II présente les mêmes corrélations que précédemment mais après une rotation de notre espace vers celui de Krumhansl.

Tableau II. Corrélations entre l'espace de Krumhansl et l'espace en 3 dimensions avec spécificités après une rotation procrustienne avec l'espace de Krumhansl. * $\mathrm{p}<.05$

\begin{tabular}{|c|c|c|c|}
\cline { 2 - 4 } \multicolumn{1}{c|}{} & $\operatorname{dim~1}$ & $\operatorname{dim~2}$ & $\operatorname{dim}$ 3 \\
\hline KWW 1 Attaque & $.98^{*}$ & -.266 & -.029 \\
\hline KWW 2 Flux & -.274 & .442 & .128 \\
\hline KWW 3 Brillance & -.033 & .141 & $.946^{*}$ \\
\hline
\end{tabular}

Après une rotation procrustienne une des trois dimensions de notre espace ne s'explique toujours pas selon l'espace de Krumhansl.

\subsection{Analyse des spécificités}

Le clavecin présente un grand poids de spécificités, ce qui est sans doute dû au résultat sonore de la mécanique spécifique de cet instrument (retour du sautereau). De même, le timbre de la clarinette présente une caractéristique qui lui est spécifique sans doute lié au rapport de ces harmoniques paires et impaires. En revanche, la guitare par exemple ne présente aucune spécificité.

\subsection{Analyses des classes}

Aucun lien entre la répartition des sujets dans les 5 classes et leur formation musicale n'est observé. En revanche, les classes se distinguent par le poids accordé sur chaque dimension et spécificités. Le Tableau III présente les pondérations des 5 classes pour les 3 dimensions et spécificités de notre espace. Ces pondérations signifient que certaines classes de sujets accordent plus d'importance à certaines dimensions perceptives. La classe 4 accorde, par exemple, le plus d'importance à la première dimension alors que la classe 5 accorde le moins d'importance à cette même dimension.

Tableau III. Pondérations des 5 classes de sujets pour les 3 dimensions et spécificités de notre espace.

\begin{tabular}{|c|c|c|c|c|}
\cline { 2 - 5 } \multicolumn{1}{c|}{} & $\operatorname{dim~1}$ & $\operatorname{dim~2}$ & $\operatorname{dim~3}$ & specif \\
\hline classe 1 & $\mathbf{1 . 1 4}$ & 0.94 & $\mathbf{1 . 1 8}$ & $\mathbf{1 . 1 7}$ \\
\hline classe 2 & 0.81 & 0.69 & 0.73 & 0.74 \\
\hline classe 3 & $\mathbf{1 . 0 5}$ & $\mathbf{1 . 7 7}$ & $\mathbf{1 . 1 2}$ & 0.58 \\
\hline classe 4 & $\mathbf{1 . 2 4}$ & 0.44 & 0.51 & $\mathbf{1 . 0 9}$ \\
\hline classe 5 & 0.76 & $\mathbf{1 . 1 5}$ & $\mathbf{1 . 3 6}$ & 0.88 \\
\hline
\end{tabular}

La classe 1 pondère plus les 3 dimensions et spécificités que la classe 2 . La classe 3 se comporte comme la première exceptée pour les spécificités. Lá classe 4 ne tient compte que de la dimension 1 et des spécificités. La classe 5 s'appuie plus sur les dimensions 2 et 3. 


\section{DISCUSSION}

Deux de nos dimensions de part leurs conrélations avec celles de l'espace de Krumhansl [2] semblent bien correspondre à ce qu'elle a nommé "qualité d'attaque" et "brillance". Ces dernières se retrouvent dans toutes les études multidimensionnelles du timbre. Les instruments se répartissent ainsi le long de la dimension "qualité d'attaque" selon deux types d'instruments; les vents et les cordes frottées à une extrémité et les cordes pincées ou barres frappées à l'autre extrémité. La dimension "brillance" répartie les instruments les plus brillants à une extrémité (ex: clavecin) et les moins brillants à l'autre extrémité (ex: clarinette). Néanmoins, il reste une 3ème dimension encore difficilement interprétable mais qui pourtant semble s'avérer pertinente dans la détermination de l'évaluation perceptive des relations entretenues par différents timbres. La nature de cette dimension a toujours posé problème dans les diverses études précédentes. Des analyses acoustiques sembleraient susceptibles de définir les corrélats physiques de cette dernière dimension (Krimphoff, 1993) bien qu'il soit difficile de cemer à l'écoute une variation perceptive systématique le long de celle-ci.

L'analyse des spécificités suggèrent que l'hypothèse des seules dimensions continues sous-tendant la structure mentale du timbre ne suffise pas. Les auditeurs s'appuieraient également sur des traits discrets dans leur jugements de dissemblance perceptive. Il est probable que la mécanique spécifique d'un instrument donné, ou encore des paramètres liés au signal acoustique, expliqueraient ces spécificités. Il reste néanmoins à effectuer des analyses acoustiques afin de mieux comprendre la nature de ces dernières. Selon certains auteurs, le degré de similarité serait défini par une fonction d'appariement des traits communs et distinctifs des 2 objets comparés [4] ou encore selon une classe commune et un univers [5]. Ces derniers modèles s'opposent aux modèles géométriques dans la mesure où la similarité perceptive de 2 stimuli serait basée. sur un processus de catégorisation, ce qui s'oppose à l'hypothèse de dimensions continues le long desquelles le degré de dissemblance ou similarité des stimuli varierait.

Par ailleurs, l'analyse CLASCAL nous a permis de dégager 5 classes latentes de sujets. La majorité des sujets se regroupant dans les 2 premières classes présentent deux stratégies bien distinctes. La première classe pondérait dans l'ensemble plus que la seconde les 3 dimensions et spécificités. L'expérience musicale des sujets ne semble cependant pas pouvoir expliquer cette différence.

\section{REFERENCES}

[1] Plomp R. Timbre as a multidimensional attribute of complex tones. In R.Plomp, G.F. Smoorenburg (Eds.), Frequency Analysis and Periodicity Detection in Hearing (1970) pp.397-414.Leiden: Sijthoff.

[2] Krumhansl C.L. Why is musical timbre so hard to understand ? In S. Nielzén \& O. Olsson (Eds.), Structure and Perception of Electroacoustic Sound and Music (1989) pp.43-53. Amsterdam: Elsevier (Excerpta Medica 846).

[3] Winsberg S. and de Soete G. A latent class approach to fitting the weighted euclidean model, CLASCAL Psychometrika 58 (1993) 315-330.

[4] Tversky, A. Features of Similarity Psychological Review 84 (1977) 327-352.

[5] Sjoberg, L. A Classificatory Theory of Similarity Psychological Research 40 (1979) 223-247. 